\title{
PD-1 Antitumor Immunity against Murine H22 Hepatocarcinoma In vivo
}

\author{
Leilei Liang ${ }^{1}$, Fuping Guan ${ }^{1}$, Yinlin $\mathrm{Ge}^{1}$
}

\begin{abstract}
${ }^{1}$ Department of Biochemistry and Molecular Biology, Medical College, Qingdao University, Qingdao, 266000, Shandong, China
\end{abstract}

\begin{abstract}
Objective: To explore the role of $P D-1$ expression level on the growth of hepatocarcinoma line H22. Methods: Tumor-bearing mice model was established with H22 cells in ICR mice. The model mice were randomly divided into two groups, control group and PD-1 interference group (PD-1-siRNA). The control group was injected transfection reagent, wherase the PD-1-siRNA was given the transfection reagent with PD-1-siRNA. Observed the life condition and tumor growth of mice, measured and recorded the tumor volume. The mRNA expression levels of $P D-L 1, P D-L 2, P 53$, caspase-3 and $I L-6$ in tumor tissue were detected by real time fluorescence quantitative PCR (qPCR) technique. The expression of IFN- $\gamma$ cytokines in spleen and tumor tissue was detected by ELISA. The ratio of Bax and Bcl-2 protein was detected by Western Blot to analyze the effect of PD-1-siRNA on tumor cell apoptosis. Results: Compared with control group, mice of PD-1-siRNA in better quality of life, survival time prolonged and the tumor volume of mouse was significantly reduced. The mRNA expression levels of $P D-L 1, P D-L 2, P 53$ and caspase -3 were increased, others, $I L-6$ expression level was significantly decreased. The expression level of IFN- $\gamma$ was up-regulated in spleen and tumor tissues. Western blot shown that the ratio of Bax and Bcl-2 was significantly increased. Conclusion: Interfere of $P D-1$ expression can effectively inhibit the growth of hepatocarcinoma cell H22 in mice.
\end{abstract}

Keywords: PD-1-siPD-1; PD-L1; Immunotherapy; H22 hepatoma cells; IFN- $\gamma$

\section{Introduction}

Liver cancer is one of the most common cancers with high incidence in the worldwide. About 370,000 people are suffering from liver cancer each year. Liver cancer with a high mortality rate, a high degree of malignancy and rapid progression ${ }^{[1]}$, makes it difficult to cure. Clinical studies have confirmed that biological immunotherapy technology ${ }^{[2 ; 3]}$ is the latest treatment of liver cancer, and it will become one of the most advantageous and promising biotechnology. The principle of biological immunotherapy is to use the body's autoimmune cells to kill tumor cells and inhibit the proliferation of tumor cells, as well as to enhance the body's immune function at the same time.

PD-1 is an important negative immune checkpoint expressed on $\mathrm{T}$ lymphocytes and plays an important role in the immune escape of tumor cells ${ }^{[4]}$. PD-1 ligands are PD-L1 and PD-L2. PD-1 binds to its ligand can inhibit the viability of $\mathrm{T}$ cells ${ }^{[5]}$. Therefore, blocking PD-1/PD-L1 signaling way by down-regulation of PD-1 can improve the vitality of T lymphocytes ${ }^{[6]}$. Varieties of studies have shown that under-regulated the expression of PD-1 helps to promote lymphocyte immune response to tumors ${ }^{[7 ; 8]}$.

In this experiment, the double-stranded siRNA PD-1 was injected into the tumor of the model mice by RNAi technique to observe its inhibitory effect on the proliferation of $\mathrm{H} 22$ cells.

\section{Experimental material and method}

2.1 H22 hepatocarcinoma xenograft tumor establishment in mice

SPF male ICR mice weighing ranging from 22 to $27 \mathrm{~g}$ were purchased from Vital River Laboratory Animal Technology co, Ltd (Beijing, China). The H22 cell line were offered by Weifang Medical College. All the animal experimental procedures in this study were conducted according to protocols approved by the institutional ethical committee of Qingdao Medical University.

H22 cells were inoculated into the abdominal cavity of ICR mice and passaged 3 times in succession. Ascite grown to 7-9 days later, extracted the vigorous ascite to 
cell count, and diluted to the concentration of $1 \times 10^{7} / \mathrm{ml}$ with PBS. Each of 20 ICR mice was inoculated with $0.2 \mathrm{ml}$ of the cell suspension into the right forelimb armpit.

\subsection{Tumor suppression effect}

The tumor bearing mice were divided into control and PD-1-siRNA after the tumor grown for 6 days. Each group was 12, and the volume of tumor was basically the same in each group. The control group was given transfection reagent (Entranster TM-in vivo, Engreen Biosystem co, Ltd) with negative control siRNA. PD-1-siRNA injected transfection reagent with PD-1-siRNA, and each mouse was administered 19.8 $\mu \mathrm{g}$. All siRNAs were synthesized by Shanghai Gemma. Tumor was injected every 3 days for a month.

The sequence of PD-1-siRNA: sense: 5'-CCUGGAGACCUCAACAAGdTdT-3', antisense: 5'-UCUUGUUGAGGUCUCCAGGdTdT-3'. The sequence of negative control RNA:sense:5'-UUCUCCGAACGUGUCACGUdTdT-3

\section{', antisense:5'-ACGUGACACGUUCGGAGAAdTdT-3'}

Medications are tumor-side injection. The longest diameter (a) and the shortest diameter (b) of the tumors were measured every 3 days, calculated tumor volume (V) by $a \times b^{2} / 2$ formulation. Observed two groups of tumor growth and draw the tumor growth curve.

\subsection{Real time fluorescence quantitative PCR assay}

Total RNA was isolated using Trizol reagent (Takara, China) from tumor tissue of the tumor bearing mice. Reverse transcription ( TransScript One-step gDNA Removal and cDNA Synthesis SuperMix, China)was performed with $4 \mu \mathrm{g}$ RNA in $20 \mu \mathrm{l}$ system. After reverse transcription the cDNA was subjected to PCR amplification (TransStart Tip Green qPCR SuperMix, China) : $94^{\circ} \mathrm{C}$ for $30 \mathrm{~s}, 94^{\circ} \mathrm{C}$ for $5 \mathrm{~s}, 60^{\circ} \mathrm{C}$ for $30 \mathrm{~s}$. Forty cycles of amplification, each sample was set to 3 parallel reaction wells, used $2^{-} \Delta \Delta^{\mathrm{Ct}}$ method to calculate the expression of the mRNA in each group. The primer sequences are shown in Table 1.

Table 1 primer sequence

\begin{tabular}{cl}
\hline gene symbol & \multicolumn{1}{c}{ primer sequence } \\
\hline$\beta$-actin & Sense: 5'-ATGGGTCAGAAGGACTCCTATG-3' \\
& Antisense:5'-ATCTCCTGCTCGAAGTCTAGAG-3' \\
PD-L1 & Sense: 5'-GGAATTGTCTCAGAATGGTC-3' \\
& Antisense: 5'-GTAGTTGCTTCTAGGAAGGAG-3' \\
PD-L2 & Sense: 5'-AAGACTGACAATCTTCCCTC-3' \\
& Antisense: 5'-CCTGAAAGTCATTAGGAGCC-3' \\
P53 & Sense: 5'-GTACCGTATGAGCCACCTGAG -3' \\
& Antisense: 5'-CGTCCCAGAAGATTCCCAC -3' \\
Caspase-3 & Sense: 5'-CTGGACTGCGGTATTGAG -3' \\
& Antisense: 5'-CGGGTGCGGTAGAGTAAGC -3' \\
IL-6 & Sense: 5'-CCACTGCCTTCCCTACTTCA -3' \\
& Antisense: 5'-AACGGAACTCCAGAAGACCA -3'
\end{tabular}

\subsection{ELISA measurements of IFN- $\gamma$ in tumor and spleen}

The 50mg tissues were cut into the RIPA lysate which contained 1\% PMSF to extract protein from the homogenate. The BCA method was used to determine the concentration of the protein, and the concentration of the protein was adjusted according to the instruction of the ELISA kit ( RayBio Mouse IFN-gama ELISA Kit, USA). Levels of IFN- $\gamma$ in tumor and spleen were measured by enzyme linked immunosorbent assays (ELISA) kits in triplicate according to manufacturer's recommended protocol.

\subsection{Western Blot}

Xenograft tumor tissues were lysed on ice in RIPA lysis buffer for $10 \mathrm{~min}$. The lysates were centrifuged at $12,000 \mathrm{~g}$ for $10 \mathrm{~min}$ at $4^{\circ} \mathrm{C}$ and supernatants were collected. The proteins were quantified with a BCA
Protein Assay Kit. Equal amount of proteins (40 mg) was loaded onto $12 \%$ SDS-PAGE and transferred to PVDF membranes. The membranes were blocked in $5 \%$ non-fat dried milk buffered for $1 \mathrm{~h}$ and incubated with primary antibodies against Bax (Santa Cruz, CA, USA) (1:400), Bcl-2 (Santa Cruz, CA, USA) (1:400) and $\beta$-actin (zhongshan, China) (1:1000) overnight at $4^{\circ} \mathrm{C}$. Then the membranes were washed and incubated with secondary antibody (MultiSciences, china) for $2 \mathrm{~h}$ at room temperature. Blots were washed three times with TBST. Through the development of ECL hypersensitive luminescent liquid (chemiluminescent HRP Subsrate), the gray value of the gel image was analyzed by software. The relative expression of the target protein was analyzed according to the ratio of $\mathrm{Bax}$ and $\mathrm{Bcl}-2$ protein gray value. 


\subsection{Statistical analysis}

The data were presented as mean \pm SD. Statistical analysis was performed with GraphPad Prism 6.0 statistical software. The significance of the data was determined by one-way analysis of variance (ANOVA) except for survival rate determined by Log-rank test and $\mathrm{P}<0.05$ was considered statistically significant.

\section{Results}

\subsection{Physiological observation}

After using PD-1 interference RNA, mice in
PD-1-siRNA group had good nutritional status, slowed tumor growth and survival time prolonged. In contrast, mice in control group gradually weakened, poor nutrition, the latter was cachexia and apperence of death in mice. As shown in figure A and B, the weight and volume of the tumor were significantly decrased than those of the control group, $\mathrm{P}<0.05$. As shown in Figure $\mathrm{C}$, the survival rate of mice was improved after PD-1-siRNA treatment compared with the control group, $\mathrm{P}<0.05$.
A

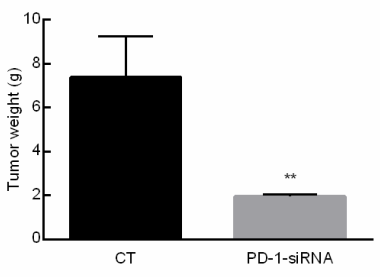

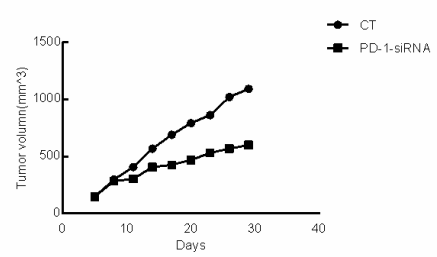

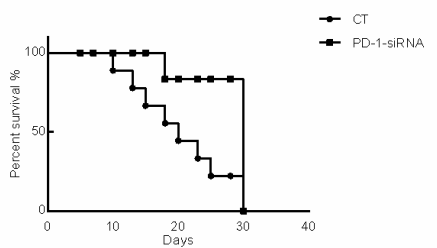

Figure1 Antitumor effect of PD-1 siRNA in the H22 xenografts. A. The weight of tumor. B. The volumn of tumor. Tumor diameters were measured at a regular interval with calipers, and the tumor volume was calculated. Compared with the control group, the weight and volumn of tumors were significantly decrased $(* * \mathrm{P}<0.01)$. C. Survival rate of mice in two groups.

\subsection{QPCR assay results}

QPCR technique was used to detect the changes in mRNA expression levels of $P D-L 1, P D-L 2, P 53$, Casepase-3 and $I L-6$ after using PD-1-siRNA. The expression levels of $P D-L 1, \quad P D-L 2, \quad P 53$ and Casepase-3 were significantly increased in tumor, While the expression level of $I L-6$ was decreased, $\mathrm{P}<0.05$.
A
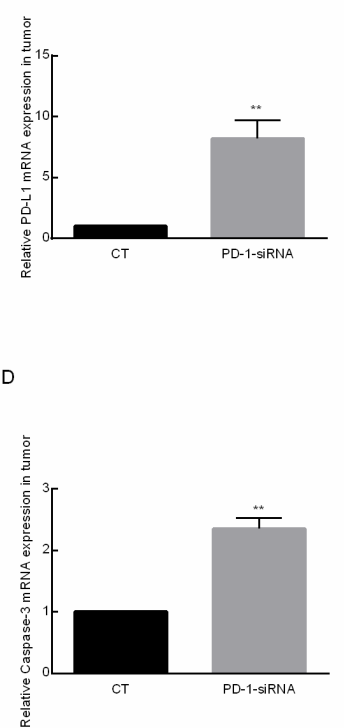

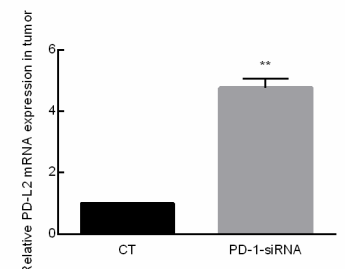

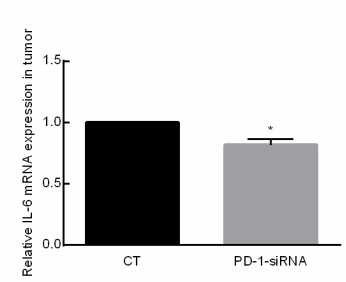

C

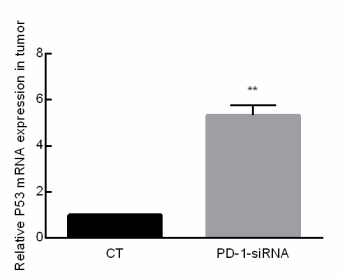

Figure 2 A. Relative $P D-L 1$ mRNA expression in tumor. B. Relative $P D-L 2$ mRNA expression in tumor. C. Relative P53 mRNA expression in tumor. D. Relative Caspase-3 mRNA expression in tumor. E. Relative IL-6 mRNA expression in tumor. $(* * \mathrm{P}<0.01, * \mathrm{P}<0.05)$ 


\subsection{ELISA assay results}

The expression levels of IFN- $\gamma$ in tumor and spleen were detected by ELISA. Compared with control group,

A

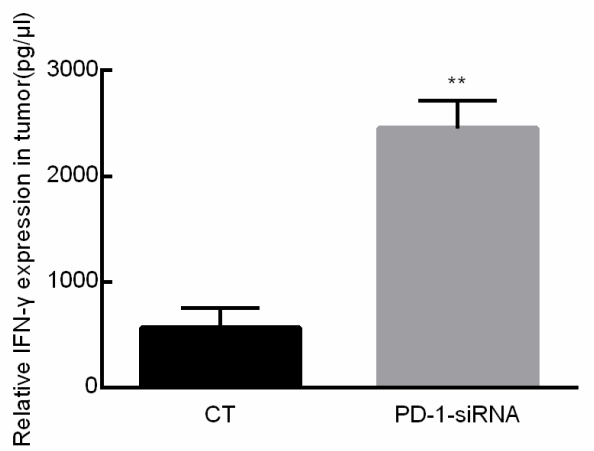

the levels of IFN- $\gamma$ was increased in tumor and spleen, $\mathrm{P}<0.05$.

B

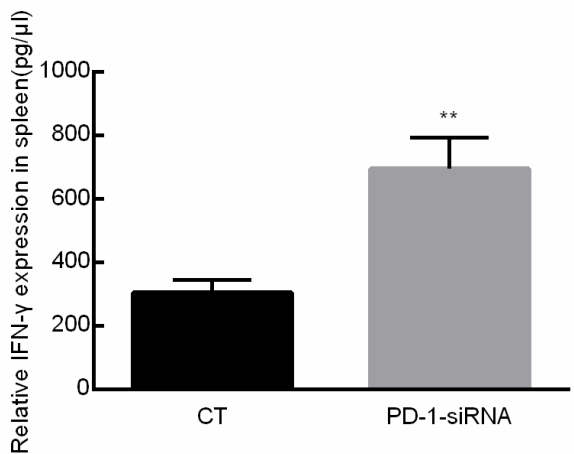

Figure 3A. Relative IFN- $\gamma$ expression in tumor. B. Relative IFN- $\gamma$ expression in spleen. $(* * \mathrm{P}<0.01)$

\subsection{Western Blot result}

The expressions of Bax and Bcl-2 protein analyzed by western blot. Results shown are representative of two independent experiments. Results were expressed as the ratio of expression level of Bax over Bcl-2. The PD-1 siRNA transfection increased the Bax/Bcl-2 ratio, $\mathrm{P}<0.05$.
A

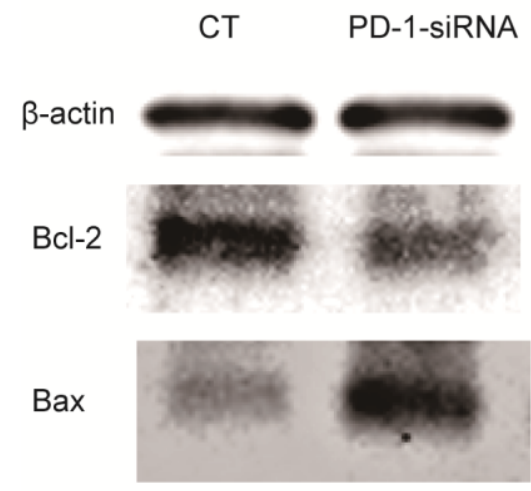

B
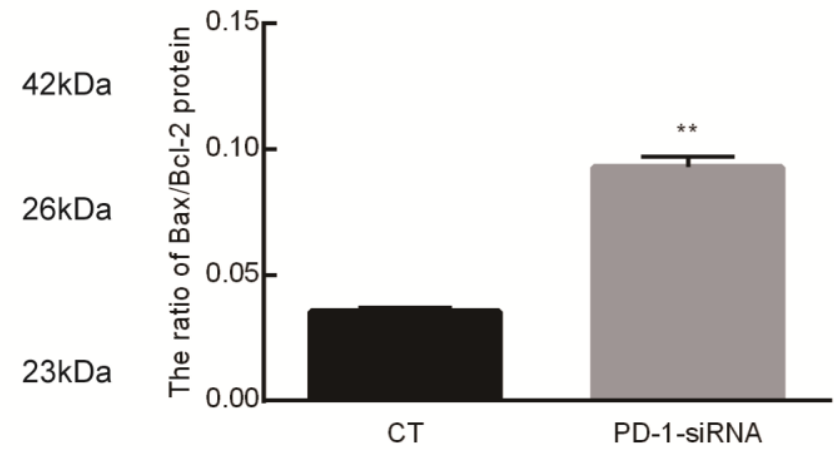

Figure 4 A. the protein levels of Bcl-2 and Bax were detected using western blot. B. The ratio of Bax over Bcl-2 protein. $(* * \mathrm{P}<0.01)$

\section{Discusstion}

$P D-1$ (programmed death receptor 1) is one of the most widely studied and used molecule which negatively regulate immunological checkpoint ${ }^{[2 ; \underline{10]}}$. It is a type I transmembrane protein weighted $55 \mathrm{kDa}$. The biological structure of PD-1 consists of extracellular domain, hydrophobic transmembrane region and cytoplasmic region. The most notable feature is that PD-1 contains two tyrosine residues located in the tail of cytoplasmic region, which contains ITIM and ITSM. ITSM is the key function area. The PD-1 located on T cell surface binds to PD-L1 and PD-L2 ligands existed on the surface of APC cells contributes to the formation of PD-1 inhibitory pathway which cause the dephosphorylation of downstream effector molecules ${ }^{[11]}$. PD-1 can also inhibit the downstream $\mathrm{NF}-\kappa \mathrm{B}$ transcription, reduce the secretion of IFN- $\gamma$ and ultimately inhibit $\mathrm{T}$ lymphocyte immunity. To a certain extent, PD-1 mediated the immune escape of tumor cells ${ }^{[12]}$. In view of the theoretical basis above, immunomodulation targeted PD-1 is of great importance in the treatment of tumors.

In this experiment, the tumor-bearing mice were divided into two groups: control group and PD-1-siRNA group. The control group was given the transfection reagent, and the PD-1-siRNA group was given the transfection reagent with interference RNA $P D-1$ for testing. It was found that PD-1 significantly inhibited and slowed down the growth of tumor. Due to 
the downregulation of PD-1 expression caused by PD-1-siRNA, the expression of its ligands $P D-L 1$ and $P D$ - L2 increased. And after using PD-1-siRNA, a variety of inflammatory cytokine secretion changes. After PD-1-siRNA was injected into the side of tumor, PD-1 activation was blocked, $\mathrm{T}$ cell viability was activated and the secretion of IFN- $\gamma$ in spleen was increased. And the IFN- $\gamma$ has multiple antitumor effects ${ }^{[13]}$, the expression of IFN- $\gamma$ in the tumor tissue also increased which can promot apoptosis of tumor cells. In contrast, the amount of $I L-6$ expression was significantly reduced in tumor. $I L-6$, as a multifunctional inflammatory cytokine, plays an important role in inflammation and tumorigenesis. IL-6 can promote the formation of tumor blood vessels ${ }^{[14]}$, which are conducive to the growth and metastasis of cancer cells and are able to suppress the immunity of the body ,mediate gene expression of many cytokines such as Bcl-x and Bcl-2, and induce EMT in tumor cells. $I L-6$ can also promote the invasiveness and distant metastasis of tumor cells ${ }^{[15]}$, and increase the degree of malignancy of the tumor. After using of PD-1-siRNA, the expression of IL- 6 mRNA decreased, which enhance the body's lethality of tumor cells.

P53 is a crucial tumor suppressor gene ${ }^{[16]}$. P53 gene inactivation plays an important role in tumor formation. P53 wild-type can cause apoptosis of cancer cells and thus prevent cancer. It also has the function of assisting gene repairing. P53 can not only regulate the expression of a variety of genes, but also participates in the regulation of some important cells signaling pathways ${ }^{[17]}$. It is also found that P53 can induce apoptosis through the activation of Caspase- 3 protein, Fuchs ${ }^{[18]}$ has found that Fas deficient cells render wild type P53, can lead to the activation of Caspase-3 protein, and induce the characteristic of apoptosis. P53-dependented apoptosis can be mediated through Fas which directly activate Caspase- 3 protein. Caspase- 3 protein belongs to the caspase protein family, and participates in the process of cell apoptosis, which is the executor of apoptotic cells ${ }^{[19]}$. The available evidence shows that the protease is closely related to apoptosis, and apoptosis may be the process of protease cascade ${ }^{[20]}$. Caspase-3 plays a very important role in triggering the caspase in the caspase cascade. Caspase-3 is activated by a variety of factors to induce hepatoma cell apoptosis, promote caspase-3 activation and apoptosis of hepatoma cells indicating caspase- 3 plays a very important role in the process of hepatoma cell apoptosis.

Bax and Bcl-2 are a pair of important apoptosis related protein in the body. The increase of Bax or decrease of the Bcl-2 can promote tumor cell apoptosis, so $\mathrm{Bax} / \mathrm{Bcl}-2$ has a closely relationship to the occurrence and development of tumor.
The general treatments of liver cancer are surgical treatment, radiotherapy and chemotherapy ${ }^{[21]}$.Although these methods can effectively inhibit the growth of hepatoma cells, it has tremendous side effects and harm to the body. Whats more, liver cancer is often diagnosed at its final stage, which made it very difficult to cure. Current research has found that biological immunotherapy has a new breakthrough in the treatment of cancer ${ }^{[22]}$. On the market there have been many drugs which block PD-1/PD-L1, which most are antibodies, although these drugs can inhibit the growth of a variety of malignant tumors, yet still they produce side effects and antibodies, which lead to immune tolerance, thus reduce the effects of the drug. In the meanwhile the use of siRNA will not cause body's immune rejection. Moreover The price of these drugs is more competitive compared with antibody drugs and may soon become a new tool for the treatment of cancer.

\section{References}

1. Jemal A, Bray F, Center M M, et al. Global cancer statistics. CA. Cancer J Clin[J]. Ca A Cancer Journal for Clinicians, 2011, 61(2): 69-90.

2. Ansell S M, Lesokhin A M, Borrello I, et al. PD-1 Blockade with Nivolumab in Relapsed or Refractory Hodgkin's Lymphoma[J]. The New England journal of medicine, 2015, 372(4): 311-319.

3. Swaika A, Hammond W A, Joseph R W. Current state of anti-PD-L1 and anti-PD-1 agents in cancer therapy[J]. Molecular Immunology, 2015, 67(2, Part A): 4-17.

4. Kim J M, Chen D S. Immune escape to PD-L1/PD-1 blockade: seven steps to success (or failure)[J]. Annals of Oncology, 2016, 27(8): 1492-1504.

5. Patsoukis N, Brown J, Petkova V, et al. Selective Effects of PD-1 on Akt and Ras Pathways Regulate Molecular Components of the Cell Cycle and Inhibit $\mathrm{T}$ Cell Proliferation $[\mathrm{J}] . \quad$ Science signaling, 2012, 5(230): ra46-ra46.

6. Ma W, Gilligan B M, Yuan J, et al. Current status and perspectives in translational biomarker research for PD-1/PD-L1 immune checkpoint blockade therapy[J]. Journal of Hematology \& Oncology, 2016, 9: 47.

7. Chauvin J-M, Pagliano O, Fourcade J, et al. TIGIT and PD-1 impair tumor antigen-specific CD8(+) $\mathrm{T}$ cells in melanoma patients[J]. The Journal of Clinical Investigation, 2015, 125(5): 2046-2058.

8. Smyth M J, Ngiow S F, Ribas A, et al. Combination cancer immunotherapies tailored to the tumour microenvironment[J]. Nature Reviews Clinical Oncology, 2015, 13: 143.

9. Buchbinder E I, Desai A. CTLA-4 and PD-1 Pathways: Similarities, Differences, and Implications of Their Inhibition[J]. American Journal of Clinical Oncology, 2016, 39(1): 98-106.

10. Reck M, Rodríguez-Abreu D, Robinson A G, et al. Pembrolizumab versus Chemotherapy for PD-L1-Positive Non-Small-Cell Lung Cancer[J]. New England Journal of Medicine, 2016, 375(19): 1823-1833.

11. Meng X, Huang Z, Teng F, et al. Predictive biomarkers in PD-1/PD-L1 checkpoint blockade immunotherapy[J]. Cancer Treatment Reviews, 2015, 41(10): 868-876.

12. Eyre T A, Collins G P. Immune checkpoint inhibition in 
lymphoid disease $[\mathrm{J}]$. British Journal of Haematology, 2015, 170(3): 291-304.

13. Shih K-S, Lin C-C, Hung H-F, et al. One-Step Chromatographic Purification of Helicobacter pylori Neutrophil-Activating Protein Expressed in Bacillus subtilis[J]. PLoS ONE, 2013, 8(4): e60786.

14. Rusolo F, Pucci B, Colonna G, et al. Evaluation of Selenite Effects on Selenoproteins and Cytokinome in Human Hepatoma Cell Lines[J]. Molecules, 2013, 18(3): 2549.

15. Park H J, Kusnadi A, Lee E-J, et al. Tumor-infiltrating regulatory $\mathrm{T}$ cells delineated by upregulation of PD-1 and inhibitory receptors $[\mathrm{J}]$. Cellular Immunology, 2012, 278(1): 76-83.

16. Jansson M D, Damas N D, Lees M, et al. miR-339-5p regulates the p53 tumor-suppressor pathway by targeting MDM2[J]. Oncogene, 2014, 34: 1908.

17. Ramani P, Nash R, Rogers C A. Aurora kinase A is superior to Ki67 as a prognostic indicator of survival in
neuroblastoma[J]. Histopathology, 2015, 66(3): 370-379.

18. Fuchs E J, Mckenna K A, Bedi A. p53-dependent DNA Damage-induced Apoptosis Requires Fas/APO-1-independent Activation of CPP32 $\beta[\mathrm{J}]$. Cancer Research, 1997, 57(13): 2550-2554.

19. Khalil H, Bertrand M J M, Vandenabeele $\mathrm{P}$, et al Caspase-3 and RasGAP: a stress-sensing survival/demise switch[J]. Trends in Cell Biology, 2014, 24(2): 83-89.

20. Steller H. Mechanisms and genes of cellular suicide[J]. Science, 1995, 267(5203): 1445-1449.

21. Tagliamonte M, Petrizzo A, Tornesello M L, et al Combinatorial immunotherapy strategies for hepatocellular carcinoma[J]. Current Opinion in Immunology, 2016, 39: 103-113.

22. Mikhail S, Cosgrove D, Zeidan A. Hepatocellular carcinoma: systemic therapies and future perspectives[J] Expert Review of Anticancer Therapy, 2014, 14(10): 1205-1218. 